\title{
Promoting Question-Asking Initiations to Peers in Young Children with Autism Spectrum Disorder (ASD) Using Video-Based Instruction
}

\section{Lema Kabashi* and Epstein AM}

Educational Studies, University of Wisconsin-La Crosse, La Crosse, Wisconsin, United States

${ }^{*}$ Corresponding author: Lema Kabashi, Educational Studies, University of Wisconsin-La Crosse, La Crosse, Wisconsin, United States, Tel: 6087858143; E-mail: Ikabashi@uwlax.edu

Received date: Sep 20, 2017; Accepted date: Oct 12, 2017; Published date: Oct 16, 2017

Citation: Kabashi L and Epstein AM (2017) Promoting Question-Asking Initiations to Peers in Young Children with Autism Spectrum Disorder (ASD) Using Video-Based Instruction. J Child Dev Disord. Vol 3.No 4: 17.

Copyright: (c) 2017 Kabashi L, et al. This is an open-access article distributed under the terms of the Creative Commons Attribution License, which permits unrestricted use, distribution, and reproduction in any medium, provided the original author and source are credited.

\section{Abstract}

The aim of this study was to promote question-asking initiations of young children with Autism Spectrum Disorder (ASD). Participants in this study were two boys, a first grader and a kindergartner. A multiple baseline single subject study design explored the efficacy of video selfmodeling with video feedback delivered via iPads. New video vignettes of participants displaying the target behavior with peers were created and utilized throughout the implementation of the intervention. Results showed an increase in appropriate question-asking initiations with peers for both participants. One of the participants maintained the skills one month after the intervention was withdrawn. Social validity findings indicated positive results related to question-asking initiations as well as other social skills.

Keywords:

Autism;Young children; Asking questions;Video selfmodeling;Video feedback

\section{Introduction}

Children with Autism Spectrum Disorder (ASD) exhibit significant deficits in age-appropriate social and communication skills, in addition to restricted and repetitive behaviors, interests, or activities (APA, 2013). Communication skills range widely, from non-verbal gestures and eye gazes to highly specified, detailed vocabularies. While some children with autism do not show any language delays (i.e., Asperger Syndrome), about 25\% may never develop functional speech during their lifetime [1].

Impairments in social and communication skills manifest in various areas such as initiating and responding to social bids, maintaining a conversation, turn taking, and establishing joint attention. Question-asking initiations are critical skills in order to initiate and maintain social interactions, be exposed to various social reinforcers, obtain novel information, and enhance independence [2]. While typically developing children may ask endless questions about people or their surroundings, children with autism tend to use language to make requests or protests rather than to initiate question-asking behaviors[3]. Failing to display question-asking initiations in early years may limit children's opportunities to develop pragmatic language skills, thus profoundly impacting their overall language development [4].

Children with autism who have functional speech have been found to demonstrate an understanding of wh-questions by 54 months [5]. Jones and Schwartz found that children with high functioning autism have difficulty requesting information to maintain a conversational topic. In a comparative study of 20 children ages 3.5 to 7 with autism and 10 children the same age who were developing typically, analyses of family dinner conversations revealed that the autism group made fewer attempts (bids) to engage verbally. Confirming earlier studies [6], Jones and Schwartz found that children with autism asked fewer questions and made approximately the same number of requests ("Mommy, please give me more soy sauce") as typically developing children.

Despite the significance that question asking has in relation to conversational exchanges with others, as well as learning novel information [7], relatively little research has been directed toward strategies that promote question asking initiations in children with autism. Researchers who investigated these skills in children with autism utilized video modeling of conversational scripts [8], video modeling and social stories, feedback and self-management [9], pivotal response treatment, time delay procedure, script-fading procedure, and applied behavior analysis intervention conducted by a robot [10-12].

Video-based instruction (VBI), an evidence-based strategy, has been effectively used to teach children with autism appropriate behaviors, including social and communication skills. VBI is used to teach appropriate skills through observation of video footage of target behaviors as models for the learner [13]. For example, Charlop and Milstein created five scripted conversations for three boys with autism. Two 
familiar adults were video-taped carrying out each conversation which included three exchanges. Questions were integrated throughout conversations. After watching the videos, researchers engaged participants in replicating the conversation. All three boys could not only carry out conversations, but their question-asking initiations also increased. Scattone also implemented VBI by combining video modeling with social stories to enhance conversational skills of a young boy with Asperger Syndrome. One of the three social stories targeted unprompted questions. The social story was shown in the video, which was narrated by an adult. After the social story ended, two adults modeled the target skill in a 5minute video of turn taking during a conversation.

This research study also uses video-based instruction to improve question-asking initiations of two young children with autism. However, video self-modeling with video feedback is utilized rather than video modeling. Kabashi and Epstein found this strategy effective for improving social initiation skills of a preschooler with autism. This study expands the use of video self-modeling with video feedback to promote question-asking initiations. In particular, it attempts to answer the following research question: Does video self-modeling with video feedback help children with autism learn how to appropriately initiate asking questions with their peers? Permission to conduct this study was issued by the Institutional Review Board of the researchers' university.

\section{Methods}

\section{Participants and settings}

Two young children with autism participated in this study. The first participant attended first grade whereas the second participant was a kindergarten student. Both participants were included in the study because they met the following inclusive criteria: a) diagnosis of Autism Spectrum Disorder (ASD), b) younger than 8 years old, and c) did not independently ask questions with peers. Consent from parents of both participants as well as peers who participated as communication partners was received before the start of the study. Participating teachers also completed informed consent statements.

The first participant was a 7-year-old boy with autism. His mild to moderate diagnosis was performed at a medical center. The evaluation report on his Individualized Education Program (IEP) indicated that his expressive and receptive language skills were within the average range. His question-asking initiations were observed by the researcher using a social interaction recording data sheet. An interview with the first participant's teacher confirmed that he could ask questions with adults but he demonstrated limited or no questions when interacting with his peers.

The second participant was a 6-year old boy who also received his diagnosis through a medical center. The severity of his autism was described as within the mild to moderate range. The IEP indicated that his communication skills were weaker than expected for his age. The social interaction observation data and results of an interview with his teacher indicated he could ask questions with adults but no questions were directed toward peers at any point.

\section{Settings}

Both participants were fully included in inclusive classrooms. The study was conducted in their classrooms twice a week during their free choice activity time. Free choice occurred twice a week for 20 minutes. The first participant's classroom included a small play area in addition to other centers (e.g., reading, morning meeting, etc.) whereas the second participant's classroom was a typical kindergarten classroom (e.g., table area, blocks area, library area, etc.).

\section{Materials}

A camcorder was used to videotape each participant's video vignettes and for data collection and reliability purposes. An Apple laptop was used to edit and create the video vignettes, which were then viewed on an iPad. The video vignette was separated into eight 15 second intervals, four of them displaying the target behaviors and the other four displaying inappropriate behaviors. To assure participants' attention and maintain motivation, video vignettes were changed as soon as the participant displayed target behaviors.

To evaluate participants' self-recognition abilities, they were videotaped for a minimum of one minute and then were asked to identify people seen in the video on the iPad. They both named themselves in the video, demonstrating selfrecognition abilities. At the same time, they demonstrated the ability to attend to the video for at least one minute. Lastly, they were very interested in the iPad, which suggested that the device itself was highly motivating.

\section{Research Design}

This study utilized a multiple baseline across participants to investigate effects of the independent variable on promoting question-asking initiations for two young children with autism. The independent variable consisted of video self-modeling with video feedback delivered via an iPad. The dependent variable consisted of appropriate question-asking initiations for information, which included WH-questions, questions starting with TO BE and HAVE verbs in all tenses (for example, "Why...?", "Do you...", "Is there...?”, "Will you...?”).

Criterion performance consisted of the participants displaying at least eight unprompted question-asking initiations to peers per session for three consecutive sessions. When the first participant showed a $25 \%$ improvement on the target behavior then the intervention with the second participant started.

\section{Baseline}

Data was collected during free choice activity time for five sessions. The teacher gave a signal/cue to the whole class to start the free choice activity. The participants would freely go and play in their favorite area. No prompts were delivered 
during that time. Data was collected twice a week for 20 minutes.

\section{Video self-modeling with video feedback}

The intervention consisted of video self-modeling with video feedback. Consistent with baseline procedures, participants engaged in a 20-minute activity session during free choice time. Participants viewed a two-minute video clip on an iPad, which was separated into eight 15-second intervals, four of them displaying the target behaviors and the other four displaying inappropriate behaviors. The researcher (the second author) showed the video clip to the participants just before the activity session took place. While watching the video the researcher asked the participant to identify and/or evaluate whether the behaviors depicted in the video were appropriate or inappropriate. The researcher provided feedback in accordance to the participant's performance and then invited him to ask questions of peers while playing, as seen in the video.

\section{Maintenance}

Three maintenance sessions, which were identical to baseline sessions, were conducted 1 month after the treatment was withdrawn for one participant. He was observed for three 20-minute sessions to assess whether he maintained treatment gains over time. A maintenance phase was not conducted for the first participant due to withdrawal of the intervention.

\section{Data Collection and Analysis}

All sessions were videotaped using a video camcorder. Video recordings were used for data collection and reliability purposes. The second author was the primary data collector and an undergraduate student served as a reliability observer. The principal investigator transferred the videos from the camera to her laptop each day to monitor participants' progress, create new videos, and to assure treatment fidelity. Data was collected for both appropriate and inappropriate question-asking initiations (see Table 1).

Table 1. Appropriate and inappropriate question-asking initiations data collection.

\begin{tabular}{|c|l|}
\hline Data Collection & Description \\
\hline Appropriate Question-Asking Initiations & $\begin{array}{l}\text { Wh-questions } \\
\text { Questions starting with "to be" and forms of the verb "to have" in all tenses (i.e., "Why...?", "Do you...", "Is } \\
\text { there...?", "Will you...?"). }\end{array}$ \\
\hline Inappropriate Question-Asking Initiations & $\begin{array}{l}\text { Question-asking initiations with adults } \\
\text { Grammatically incorrect question-asking initiations with adults or peers } \\
\text { Any other verbalizations that were not questions }\end{array}$ \\
\hline
\end{tabular}

\section{Reliability}

Video recordings of the first participant's performance during choice time before the study started were used for reliability training. The operational definitions of the target behaviors and a scoring protocol were provided to the reliability observer. Initial training consisted of the reliability observer engaging in a practice session during which she identified and recorded target behaviors while watching the video recordings described above. Her recordings were compared to recordings of the principal investigator. Training continued in this fashion until $90 \%$ agreement for the target behavior was reached on three consecutive sessions. Interobserver agreement was calculated by dividing the total number of agreements by the total number of agreements plus disagreements multiplied by 100 . The reliability observer randomly selected and independently scored $33-40 \%$ of video sessions from each condition and participant. The IOA for the first participant ranged from $77 \%$ to $100 \%$ with an average of $81 \%$. As for the second participant, the IOA ranged from $66 \%$ to $100 \%$ with an average of $80 \%$.

To determine whether the intervention procedures were being implemented accurately and consistently, procedural reliability data sheets were developed for each condition. The same undergraduate student served as the treatment fidelity observer and reviewed the video recordings using a checklist to evaluate whether the intervention was implemented accurately and consistently. The reliability observer recorded "yes" or "no" if the steps were followed and implemented accurately and "N/A" if any steps were not applicable. Treatment fidelity was calculated for each session as follows: total number of steps completed accurately divided by the total number of steps completed accurately plus the total number of steps completed inaccurately/missed multiplied by 100. Treatment fidelity conducted for $33-40 \%$ of sessions across conditions was $100 \%$ for all conditions.

\section{Data analysis}

The effect of video self-modeling with video feedback on appropriate question-asking initiations with peers was determined through visual inspection of the graphic representation of the data. Appropriate question-asking initiations with peers were calculated by summing the scores of the dependent variable for each session within that condition.

\section{Social validity}

Upon completion of the study, researchers interviewed classroom teachers regarding the efficacy of video selfmodeling with feedback in developing question-asking 
initiations. Throughout the intervention, classroom teachers also shared their views regarding the efficacy of the intervention as well as the strengths and weaknesses of the intervention.

\section{Results}

The frequency of appropriate question-asking initiations with peers for both participants increased, although with different speeds and intensity. Although, as seen in Figure 1, data on the first participant's performance shows variability, it is important to note that this variability is associated with the peer who was partnered with during the activity session.

The baseline condition for the first participant, which was conducted through five sessions, showed that he displayed limited appropriate question-asking initiations with peers. Appropriate question-asking initiations with peers ranged from 0 to $4(M=2)$ while inappropriate question-asking initiations ranged from 0 to $3(M=1.4)$.

The first participant increased appropriate question-asking initiations dramatically after the intervention was introduced. The target behavior increased right away; nine appropriate question-asking initiations with peers occurred on day one of the intervention implementation. Overall, the number of question-asking initiations with peers ranged from 1 to $20(\mathrm{M}=$ 8.57) across 14 sessions. Inappropriate question-asking initiations remained low $(\mathrm{M}=0.2$; range $0-1)$.

Maintenance was conducted across three sessions where he was observed for three consecutive 20-minute sessions. Appropriate question-asking initiations ranged from 3-5 (M = 4) whereas inappropriate question-asking initiations remained low $(\mathrm{M}=1.3$; range $0-2)$.

The baseline condition for the second participant indicated very limited question-asking initiations with peers. Appropriate question-asking initiations with peers ranged from 0 to $2(M=$ 0.8 ) while inappropriate question-asking initiations ranged from 0 to $1(M=0.1)$.

The second participant showed improvements on appropriate question-asking initiations with peers during the intervention condition. However, progress was slow for the first four sessions. It wasn't until the sixth session that he displayed eight question-asking initiations with peers. His target behavior remained high compared to baseline for another three sessions, but started to drop in sessions 10 and 11. Overall the number of target behaviors ranged from $0-8$ $(\mathrm{M}=3.7)$ across 11 sessions. Due to time constraints and a drop in the second participant's target behavior, the intervention was withdrawn at session 11 . Consequently, the maintenance condition was not conducted for the second participant.

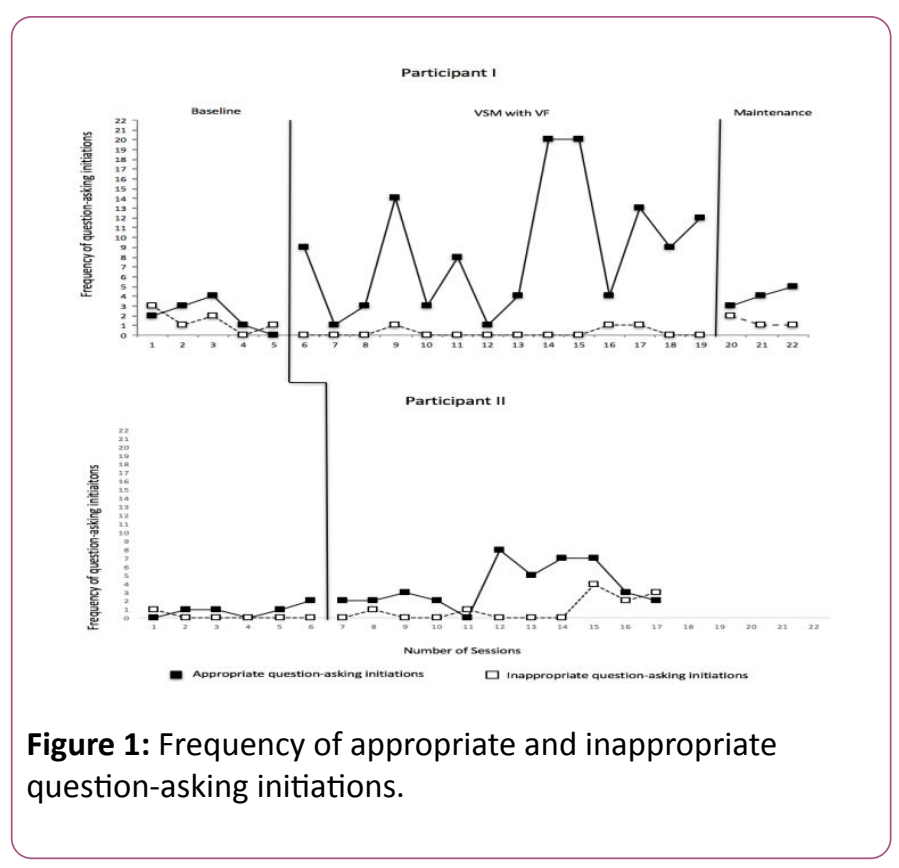

\section{Social validity}

Social validity conducted with the participants' teachers indicated that the intervention was effective for both participants, not only regarding targeted skills but also for overall social skills and communication with their peers. Both the first participant's first-grade teacher and his personal assistant completed a social validity questionnaire. They noted that the first participant asked his peers no more than four questions at baseline. His personal assistant stated that he asked peers 9 to 12 questions as a result of the intervention while his teacher stated that the first participant asked more than 17 questions. This discrepancy may be due to the first participant indeed asking more than 17 questions during one session but not routinely. Both the first participant's teacher and his personal assistant strongly agreed that he enjoyed watching his video on the iPad and that he responded appropriately to questions/feedback with the interventionist. They also strongly agreed that VSM with video feedback was easy to implement and they strongly recommended this intervention for other children with autism. Finally, they both strongly agreed that the first participant asked questions with peers in other environments and during other times of the day.

The second participant's teacher stated that he developed closer relationships with his peers during the intervention. She noted that his eye contact improved and he appeared to be more purposeful with his communications. He eagerly watched his video at the beginning of each session and asked if his friends could watch along with him. The second participant's peers were so drawn to the videos that his teacher needed to limit the number of friends allowed to watch. She noted that the intervention appeared to help the second participant understand the rather abstract notion of interacting with peer, making it "more real". 


\section{Discussion}

It was hypothesized that participants would increase appropriate question-asking initiations with peers after viewing videos of themselves asking their peers questions. Results of this study supported the hypothesis; both participants increased question-asking initiations with peers during the intervention compared to baseline. The first participant displayed a significant increase in question-asking initiations with his peers at the first intervention session, while the second participant was quite close to meeting the criteria (7 out of 8 question-asking initiations) in sessions eight and nine but failed to do so in the following session.

The first participant's graph shows variability in data during the intervention. It is important to note that when the frequency of question-asking initiations was low, the first participant initiated asking questions with his peers but once he did not receive a response then he would not continue with question-asking initiations. However, the first participant demonstrated a markedly different communication interaction with one of his peers who was recruited as a communication partner. Unfortunately, his communication partner often participated in reading and math remediation sessions during choice time and was not available to play with the first participant. Variability in the first participant's appropriate question-asking initiations during five sessions $(5,7,8,11$ and 13) coincide with days that his communication partner was not present during choice time.

Spikes in the number of questions the first participant asked when playing with his communication partner point to the importance of identifying a trusted peer. Increasing numbers of children with autism who have relatively strong verbal skills are included in general education classrooms. Establishing peer relationships is hard work for these children. As derived from the results of this study, partnering children with autism with a willing, supportive peer while they are developing language and social skills is an essential component of successful inclusion. The first participant asked question after question after question with his communication partner. It was as if once he learned how to ask questions, and he knew that he was communicating with a supportive peer, he had to keep asking questions.

The first participant's performance during the three maintenance probes supports previous research findings regarding the long-term efficacy of VSM with VF given that a high frequency of appropriate question-asking initiations with peers was maintained one month after the intervention was withdrawn. Informal data from the first participant's teacher confirmed that he consistently asked questions from the time of intervention withdrawal until the maintenance probes were conducted, and even continued for several additional weeks, thus further strengthening the efficacy of intervention.

The second participant learned how to ask questions with his peers, however he had difficulty meeting the criteria of at least eight question-asking initiations for three consecutive sessions. He seemed to focus on the quality (e.g. correct tense, appropriateness for the situation) rather than the quantity of question-asking initiations. The second participant smiled and exclaimed to the researcher, "that was a good question!" nearly every time he initiated question asking with a peer. Instead of asking additional questions with his peers he discontinued his discourse after one "good" question-asking initiation. This finding supports suggestions from Palmen et al. that question asking initiation is a performance deficit rather than a skill deficit. While the current study suggests that children with autism can learn to ask appropriate questions, other strategies may be required to help them sustain frequent meaningful question-asking interactions.

\section{Limitations}

There are a number of limitations that are associated with the present study. First, the number and length of conditions for each participant differed. While all three conditions (i.e., baseline, intervention, maintenance) were implemented for the first participant, only two conditions (i.e., baseline, intervention) were implemented for the second participant. The intervention started with the first participant and it was implemented until he met the criteria. The intervention for the second participant, however, ended before he met the criteria. This occurred for two reasons. First, he did not meet the criteria with consistency (eight target behaviors for three consistent sessions). However, he met the criteria in session six and then in sessions eight and nine he missed the criteria by only one question-asking initiation. Unfortunately, the number of questions dropped in the following sessions and he started loosing interest. Second, after session 11 the kindergarten classroom started integrating more outdoor activities to coincide with the end of the school year. These activities impacted both the implementation and the efficacy of the intervention. Additional time may have promoted the target behaviors, given that many video self-modeling interventions targeting social initiations were implemented for more than eleven sessions.

Implementation of the intervention only twice a week, especially for the second participant, appears to be a second limitation. Although his kindergarten classroom had choice time every day, the intervention was implemented two times per week to assure consistency with the first participant's classroom practice of choice time only occurring twice a week. It is well established that there is a positive relationship between treatment intensity and mastery of learning outcomes of children with autism. One might speculate that if a high-intensity intervention was implemented with the second participant the outcomes of the study could be different.

Third, the participants' classroom teachers recommended peers. While the peers were willing, their responsiveness to the participants varied. In addition, they were simply prompted to play with the participants rather than being trained. Peer-mediated interventions are strongly recommended as strategies that enhance social interactions of students with autism in schools. However, research shows positive outcomes when selected peers are interested in social interactions with children with autism and are also trained as 
peer communication partners.If participants' peers had been trained as communication partners, frequency of questionasking initiations may have increased, especially for the second participant.

Finally, slight modifications were made to help the second participant increase his number of question-asking initiations. Beginning at session five, in addition to the initial video shown before the activity session started, he viewed another 30 second video vignette of asking an appropriate question approximately every 10 minutes during the activity session. His teacher also suggested using a pipe cleaner with beads to keep track of how many questions the second participant asked. He was allowed to thread a bead onto a pipe cleaner each time he asked a question, providing tactile and visual confirmation that he was indeed making progress with his target skill. While this modification helped the second participant ask additional questions for two sessions (session 8 and 9), he then lost interest and returned to proclaiming his success with one question ("That was a good question!") and refrained from asking additional questions. These modifications did not change core components of the intervention, nor did they result in an increase in the number of question-asking initiations. The use of additional strategies (e.g., reinforcement, self-management, prompting) are very common in video-based instruction [15-24] because they do not change the core components of the intervention.

\section{Further Recommendations Conclusion}

Despite the aforementioned shortcomings of this study, there is clear evidence that children with autism can learn an important skill such as initiating question asking with peers from video self-modeling with video feedback via iPads. Both participants demonstrated an increase in this target skill in a naturalistic setting. The importance of conducting interventions in naturalistic rather than controlled settings has been noted in recent studies of children with autism [24-28]. While further research is needed to study using VSM with video feedback in various inclusive settings (e.g., playground, bus, cafeteria, community), the current study suggests promising outcomes.

Implementing VSM with VF via iPads is relatively easy because it is both portable and non-invasive. Both children enjoyed watching their videos on iPads throughout the intervention. This enjoyment appeared to extend to their peers. The kindergarten participant enthusiastically invited his peers to join him in watching his video and they responded with the same enthusiasm. Other children in the first grader's classroom also demonstrated a strong interest in the video, asking the researcher if they could watch along with the participant. Further research could investigate building friendships utilizing VSM with VF via iPads in various inclusive settings.

This study addressed a crucial and challenging component of language: question-asking initiations. Although both participants learned how to ask questions, the second participant appeared "stuck" at the level of asking one question, noting that it was "a good question", while the first participant did ask a range of questions. One of the times the second participant did ask numerous questions, he and a friend played with a role of tickets, repeatedly asking their peers, "Do you want a ticket?" Often the first participant also repeated the same question several times with either the same or a different peer. Future studies should focus on teaching children with autism how to ask questions that will lead them towards the acquisition of novel information. Although Taylor and Harris taught children with autism to seek information (specifically, "What's that?") using a time delay procedure, we recommend expanding the range of evidencebased strategies by including video self-modeling with video feedback to target question-asking initiations for novel information. For example, the second participant could expand his inquiry by asking his peers where they would like to go with their ticket, which could lead to a meaningful conversational exchange with peers.

The study's positive results suggest that video self-modeling with video feedback could be implemented in inclusive publicschool settings to assist children in developing additional language skills, such as making requests and extending and expanding peer's statements. Strengthening language skills could assist young children with autism as they interact with their peers and most importantly, eventually develop and maintain meaningful friendships.

\section{References}

1. American Psychiatric Association (2013) Diagnostic and statistical manual of mental disorders (5th ed.). Washington: American Psychiatric Association.

2. Eigsti I, Bennnetto L, Dadlani M (2007) Beyond pragmatics: Morphosyntactic development in autism. J Autism Dev Dis 37:1007-1023.

3. Bellini S, Akullian J (2007) A meta-analysis of video modeling and VSM interventions for children and adolescents with autism spectrum disorders. Excep Child 73: 264-287.

4. Bellini S, Akullian J, Hopf A (2007) Increasing social engagement in young children with autism spectrum disorders using video self-modeling. Sch Psyc Rev 36: 80-90.

5. Buggey T, Hoomes G, Sherberger ME, Williams S (2011) Facilitating social initiations of preschoolers with autism spectrum disorders using video self-modeling. Foc on Autism Dev Dis 26: 25-36.

6. Carter (2017) Efficacy of peer support interventions in general education classrooms for high school students with autism spectrum disorder. Rem Spec Edu 38: 207-221.

7. Charlop MH, Milstein JP (1989) Teaching autistic children conversational speech using video modeling. J Appl Beh Anal 22: 275-285.

8. Gelbar NW, Anderson C, McCarthy S, Buggey T (2012) Video selfmodeling as an intervention strategy for individuals with autism spectrum disorders. Psyc Sch 49: 15-22.

9. Goodwin, A., Fein, D., \& Naigles, L. (2012). Comprehension of wh-questions precedes their production in typical development and autism spectrum disorders. Autism Res5:109-123. 
10. Goodwin A, Fein D, Naigles $L$ (2015) The role of maternal input in the development of wh-question comprehension in autism and typical development. The J Child Lang 42: 32-63.

11. Gül SO, Vuran S (2010) An analysis of studies conducted video modeling in teaching social skills. Edu Sci Theo Prac 10: 249-274.

12. Hughes C (2013) Increasing conversational interactions between verbal high school students with autism and their peers without disabilities. Foc Autism Dev Dis 28: 241-254.

13. Jones CD, Schwartz IS (2009) When asking questions is not enough: An observational study of social communication differences in high functioning children with autism. J Autism Dev Dis 39: 432-443.

14. Kabashi L, Epstein A (2017) Improving social initiations of children with autism using video self-modeling with video feedback: A case study. J Edu Soc Res 7: 111-121.

15. Kabashi L, Kaczmarek LA (2017) Evaluating the efficacy of videobased instruction (vbi) on improving social initiation skills of children with autism spectrum disorder (asd): A review of literature. Rev J Autism Dev Dis 4: 61-81.

16. Kabashi L, Kaczmarek LA (2017) The efficacy of a video selfmodeling intervention on peer social initiation skills of children with autism spectrum disorders (asd). Eur J Soc Sci Edu Res 10: 305-321.

17. Leach D, Duffy ML (2009) Supporting students with autism spectrum disorders in inclusive settings. Interv Sch Clin 45: 31-37.

18. Linstead (2017) Intensity and learning outcomes in the treatment of children with autism spectrum disorder, Behav Modif 41: 229-242.

19. Marans, W. D., Rubin, E., \& Laurent, A. (2005). Addressing social communication skills in individuals with high-functioning autism and Asperger syndrome: Critical priorities in educational programming.

20. FR Volkmar, R Paul, A Klin, D Cohen (Eds.) Handbook of autism and pervasive developmental disorders (pp. 977-1002). New Jersey: John Wiley \& Sons.

21. Palmen A, Didden R, Arts $M$ (2008) Improving question asking in high-functioning adolescents with autism spectrum disorders. Autism 12: 83-98.

22. Rubin $E$, Lennon $L(2004)$ Challenges in social communication in Asperger syndrome and high-functioning autism. Top Lang Disord 24: 271-285.

23. Scattone D (2008) Enhancing the conversation skills of a boy with Asperger's disorder through social stories and video modeling. J Autism Dev Disord 38: 395- 400.

24. Seung HK (2007) Linguistic characteristics of individuals with high functioning autism and Asperger syndrome. Clin Linguist Phon 21: 247-259.

25. Smith J, Hand L, Dowrick PW (2014) Video feedforward for rapid learning of a picture-based communication system. J Autism Dev Disord 44: 926-936.

26. Taylor BA, Harris SL (1995) Teaching children with autism to seek information: Acquisition of novel information and generalization of responding. J Appl Behav Anal 28: 3-14.

27. Wong C, Odom SL, Hume KA, Cox AW, Fettig A,et al. (2015) Evidence-based practices for children, youth and young adults with autism spectrum disorder: A comprehensive review. J Autism Dev Disord 45: 1951-1966.

28. Zager D, Wehmeyer ML, Simpson R (2012) Educating students with autism spectrum disorders: Research- based principles and practices. New York, NY: Taylor \& Francis. 